\title{
Improved training of deep convolutional networks via minimum-variance regularized adaptive sampling
}

\author{
Alfonso Rojas-Domínguez ${ }^{1}$ \\ - S. Ivvan Valdez ${ }^{2}$ (iD \\ Manuel Ornelas-Rodríguez \\ - Martín Carpio ${ }^{1 \otimes(i)}$
}

Received: date / Accepted: date

\begin{abstract}
Fostered by technological and theoretical developments, deep neural networks have achieved great success in many applications, but their training by means of mini-batch stochastic gradient descent (SGD) can be very costly due to the possibly tens of millions of parameters to be optimized and the large amounts of training examples that must be processed. Said computational cost is exacerbated by the inefficiency of the uniform sampling method typically used by SGD to form the training mini-batches: since not all training examples are equally relevant for training, sampling these under a uniform distribution is far from optimal. A better strategy is to form the mini-batches by sampling the training examples under a distribution where the probability of being selected is proportional to the relevance of each individual example. This can be achieved through Importance Sampling (IS), which also achieves the minimization of the gradients' variance w.r.t. the network parameters, further improving convergence. In this paper, an IS-based adaptive sampling method is
\end{abstract}

\footnotetext{
$凶$ Martín Carpio, PhD

juanmartin.carpio@leon.tecnm.mx
}

Alfonso Rojas-Domínguez, $\mathrm{PhD}$

alfonso.rojas@gmail.com

S. Ivvan Valdez, PhD

svalddez@centrogeo.edu.mx

Manuel Ornelas-Rodríguez, PhD

manuel.ornelas@leon.tecnm.mx

1 Tecnológico Nacional de México/Instituto Tecnológico de León, 37290, León, Guanajuato, México.

2 CONACYT-Centro de Investigación en Ciencias de Información Geoespacial, CENTROGEO, A.C., C.P. 76703, Querétaro, México. studied that exploits side information to construct the required probability distribution. Said method is modified to enable its application to deep neural networks, and the improved method is dubbed Regularized Adaptive Sampling (RAS). Experimental comparison (using deep convolutional networks for classification of the MNIST and CIFAR-10 datasets) of RAS against SGD and against another sampling method in the state of the art, shows that RAS achieves relative improvements of the training process, without incurring significant overhead or affecting the accuracy of the networks.

Keywords Deep learning · Convolutional Neural Networks · Gradient Descent · Importance Sampling

\section{Introduction}

During the past decade, the increase in available data and computational power, as well as theoretical developments, propelled the dominance of Deep Neural Networks (DNNs) in applications such as computer vision, pattern classification, natural language processing, forecasting and so on (Alom et al. (2018)). However, due to the millions of trainable parameters that DNNs contain and the large amounts of data to be processed, notwithstanding the capabilities of modern computers, training of DNNs can be very costly (Wang et al. (2018)).

Because of the non-convexity of the problem, the standard method for training many types of DNNs is mini-batch gradient descent, often referred to simply as Stochastic Gradient Descent (SGD). The conventional way of forming the data mini-batches for SGD is through uniform sampling of the training data-instances, but since data instances are not equally useful, this sampling strategy is not very efficient. In recent years, 
methods to improve the training of DNNs via more efficient sampling schemes have been proposed around the idea of prioritizing the selection of instances that are most important for learning. Notice that an instance's importance constantly changes during training because the model parameters (w.r.t. which the gradients for SGD are computed) are constantly modified. Thus, the importance distribution is not static, but dynamic; it cannot be computed once/off-line and then be used throughout the training, but it must be updated on-line along with the parameters of the model. For training of DNNs this poses a computational challenge.

The importance distribution for SGD can be defined by satisfying a strongly principled optimization criterion via a more general strategy known as Importance Sampling (IS). In statistics, IS is a technique for estimating properties of a target distribution based on samples from a surrogate distribution (Tokdar and Kass (2010)). IS is also an important variance reduction technique (Owen (2013)), which makes it relevant for training via SGD because minimization of the variance of stochastic gradients within the training mini-batches leads to smoother and faster reduction of the loss function, effectively improving training (Zhao and Zhang (2014)). Thus, the optimal distribution for reduction of the gradient variance is proportional to the per-instance gradient norm (Zhao and Zhang (2015)), but computing said distribution for DNNs is unfeasible in practice because of the computational cost involved. A simple but not very satisfactory alternative is to employ the corresponding loss value as a proxy for the required gradient norm, since these quantities are loosely correlated. Another alternative is to use bounds or coarse estimates for the required gradients, which are much less expensive to compute during training. Using these alternatives (an approximation to the norm of gradients or the loss values), several selective sampling methods for training of learning models have been described in the literature (See Section 2).

The aim of this paper is to examine one of such methods, called Adaptive Sampling (AS) (Gopal (2016)) which includes interesting and comprehensible notions that have been shown to offer improvements over regular SGD but also, to the best of our knowledge, have not been tested for the training of DNNs due to scalability limitations. Furthermore, we identify a main drawback of AS and propose modifications intended to counteract it, thus producing the Regularized Adaptive Sampling (RAS) method. Our proposal is experimentally compared against the standard sampling scheme for SGD as well as against Online Batch Selection (OBS) (Loshchilov and Hutter (2015)) in the context of image classification, using the MNIST and the CIFAR10 datasets. Our results show that RAS outperforms OBS in general and SGD in some occasions while being equivalent in the rest, all of this with little overhead.

The rest of this paper is divided into six sections. Section 2 provides an overview of related work in the literature; Section 3 introduces the mathematical formulation of the selected sampling approaches; Section 4 contains the description of our proposal; Section 5 reports the results from our experimental comparison; Section 6 offers a discussion around our understanding of noteworthy results; Section 7 closes the paper.

\section{Related Work}

Several strategies to improve the convergence of SGD have been described in the literature. Herein we are interested in the methods for improving the way in which data instances are selected to form training minibatches by replacing the uniform distribution typically employed to sample training instances.

Regarding strategies built around IS: Zhao and Zhang (2015), show that the optimal importance distribution is proportional to the per-instance gradient-norm and establish a clear connection with the variance of the gradient estimates in SGD. Gopal (2016), employs side information (e.g. the classes in a classification problem) to define a distribution that is directly proportional to the norm of the gradients. Note that these two works are not directed at the training of full-scale $\mathrm{DNNs}^{1}$, whose size hinders the direct application of IS based on the per-sample gradient-norm. For full-scale DNNs, Katharopoulos and Fleuret (2017) generated the importance distribution using the loss value as an alternative importance metric that nevertheless succeeds at reducing the variance of the gradients. Later on, Katharopoulos and Fleuret (2018) derived an upper bound (16) to use in-lieu of the actual gradient-norm; this is an important contribution that we incorporate into our method.

A different, empirical approach to define importance distributions employs the loss value of individual training instances. The loss is a measure of how well a model can solve particular instances; if the loss of an instance is high then it should be sampled more frequently so that the model has more opportunities to learn to solve it. This should make the learning process more efficient. Following this approach, Loshchilov and Hutter (2015) ranked the instances w.r.t. their latest known loss value and built a probability distribution that decays exponentially as a function of that ranking; this is called

1 The term deep network may allude to small networks with relatively few parameters presented in more than one hidden layer. What we call full-scale models refers to DNNs with around a dozen layers and typically millions of parameters. 
OBS. In yet another approach inspired by how humans and animals learn, Bengio et al. (2009) presented training instances to learning models in gradually increasing amounts and degree of complexity, achieving faster convergence and better generalization in a variety of toy problems, recognition of shapes, and language models. This method is called Curriculum Learning.

Other proposals to optimize the training of DNNs can also be found in different contexts. Alain et al. (2015) explored the application of IS to Asynchronous SGD, where the required gradients are computed in parallel, and this task is distributed over a set of machines. Wu et al. (2017) designed a distribution that maximizes the diversity of the losses in a training batch and applied this idea in deep embedding learning, which is a problem of clustering instances in the so-called embedding space induced by the learning model. Fan et al. (2017) used reinforcement learning to train a neural network that selects instances in order to optimize the convergence of a second neural network. Joseph et al. (2019) designed a mini-batch selection strategy based on the maximization of a submodular function that captures relevant information from the data used to speed up the training. Finally, a review on optimization approximation and other techniques to enable large-scale machine learning, can be found in (Wang et al. (2020)).

Our RAS method presented in Section 4 is an adaptation of the AS algorithm of Gopal (2016) for DNNs, with the added improvement of a redefined probability distribution that combines a gradient-norm estimate and the loss of individual instances to form the mini-batches. This proposal is compared against uniform sampling and against OBS. Details of these methods are provided in the following section.

\section{Selective Sampling Approaches}

Consider the problem of optimizing the parameters of a deep learning model $y_{i}=\varphi\left(x_{i} ; \theta\right)$ for classification, where $\theta$ represents the model parameters, $x_{i} \in \mathbb{R}^{n}$ represents an input data instance and $y_{i}$ is its corresponding class label. Assuming a non-negative loss function $L\left(\varphi\left(x_{i} ; \theta\right), y_{i}\right)$, the standard solution $\theta^{*}$ is:

$$
\theta^{*}=\underset{\theta}{\arg \min } \mathbb{E}_{x \sim \mathcal{U}} L(\varphi(x ; \theta), y)
$$

where $\mathcal{U}$ represents the uniform distribution and, given a dataset $D=\left\{\left(x_{1}, y_{1}\right),\left(x_{2}, y_{2}\right), \ldots,\left(x_{N}, y_{N}\right)\right\},(1)$ can be iteratively estimated via the SGD algorithm.

As discussed in previous sections, employing a nonuniform distribution for the selection of the training instances can make the process of solving (1) more efficient through two main approaches: constructing the sampling distribution with base on the values of $L(\varphi, y)$, or with base on the gradients $\nabla_{\theta} L(\varphi, y)$. This should be integrated along the standard training procedure that presents the training data to the learning model in minibatches (or simply, batches) of convenient size.

Online Batch Selection: Following the first approach, Loshchilov and Hutter (2015) proposed to sort $\left\{x_{i}\right\}_{i=1}^{N}$ in descending order w.r.t. $\left\{L\left(\varphi\left(x_{i} ; \theta\right), y_{i}\right)\right\}_{i=1}^{N}$ and assign to the $j$-th ranked data instance a probability $p_{j}$ defined as:

$$
p_{j}=\frac{\exp \left(\log \left(s_{t}\right) / N\right)^{-j}}{\sum_{i=1}^{N} \exp \left(\log \left(s_{t}\right) / N\right)^{-j}}
$$

where $\exp \left(\log \left(s_{t}\right) / N\right)$ is the probability drop between consecutive instances and $s_{t}=p_{1} / p_{N}$ at iteration $t$ acts as a selection-pressure parameter that can be kept constant during training or may be decreased over time.

Using (2), Loshchilov and Hutter designed a lossdependent instance-selection method called OBS, and showed that it can accelerate the training of a Convolutional Neural Network (CNN). OBS is an appealing proposal because it can be implemented very easily over a learning model as a wrapper function with little overhead. In this work, RAS is compared against OBS.

Adaptive Sampling: Following the second approach, Gopal (2016) developed an instance-selection method called AS wich integrates Importance Sampling for minimization of the gradient variance with respect to a non-uniform probability distribution defined over a partition $C$ of the dataset $D$, generated with base on side information, such as the class labels, $y$.

Briefly explained, $C=\left\{c_{1}, c_{2}, \ldots, c_{k}\right\}$ and each bin $c_{j}=\left\{x_{1}^{j}, x_{2}^{j}, \ldots, x_{m_{j}}^{j}\right\}, \forall j=1, \ldots, k$. In our notation a superindex is used to indicate the $j$-th bin and a subindex indicates the $i$-th element of the corresponding $j$-th bin. That is, the $N$ instances are split into $k$ mutually exclusive not necessarily balanced bins. The probability of selecting the $i$-th instance is:

$$
P\left(x_{i}\right)=p_{j} \frac{1}{\left|c_{j}\right|}
$$

where $p_{j}=\mathcal{P}\left(C=c_{j}\right)$ is the probability of selecting the $j$-th bin, whose selection should reduce the variance $V$ of the descent direction, posing the problem:

$$
\min _{P} V\left[d^{t}\right]=\min _{p_{1}, \ldots, p_{k}}\left(\mathbb{E}\left[d^{t^{\mathrm{T}}} d^{t}\right]-\mathbb{E}\left[d^{t}\right]^{\mathrm{T}} \mathbb{E}\left[d^{t}\right]\right)
$$

where $d^{t}$ is the descent direction at iteration $t$ :

$$
d^{t}=\frac{1}{N} \frac{1}{P\left(x_{i}^{t}\right)} \nabla_{\theta} L\left(\varphi\left(x_{i}^{t} ; \theta^{(t-1)}\right), y_{i}\right) .
$$



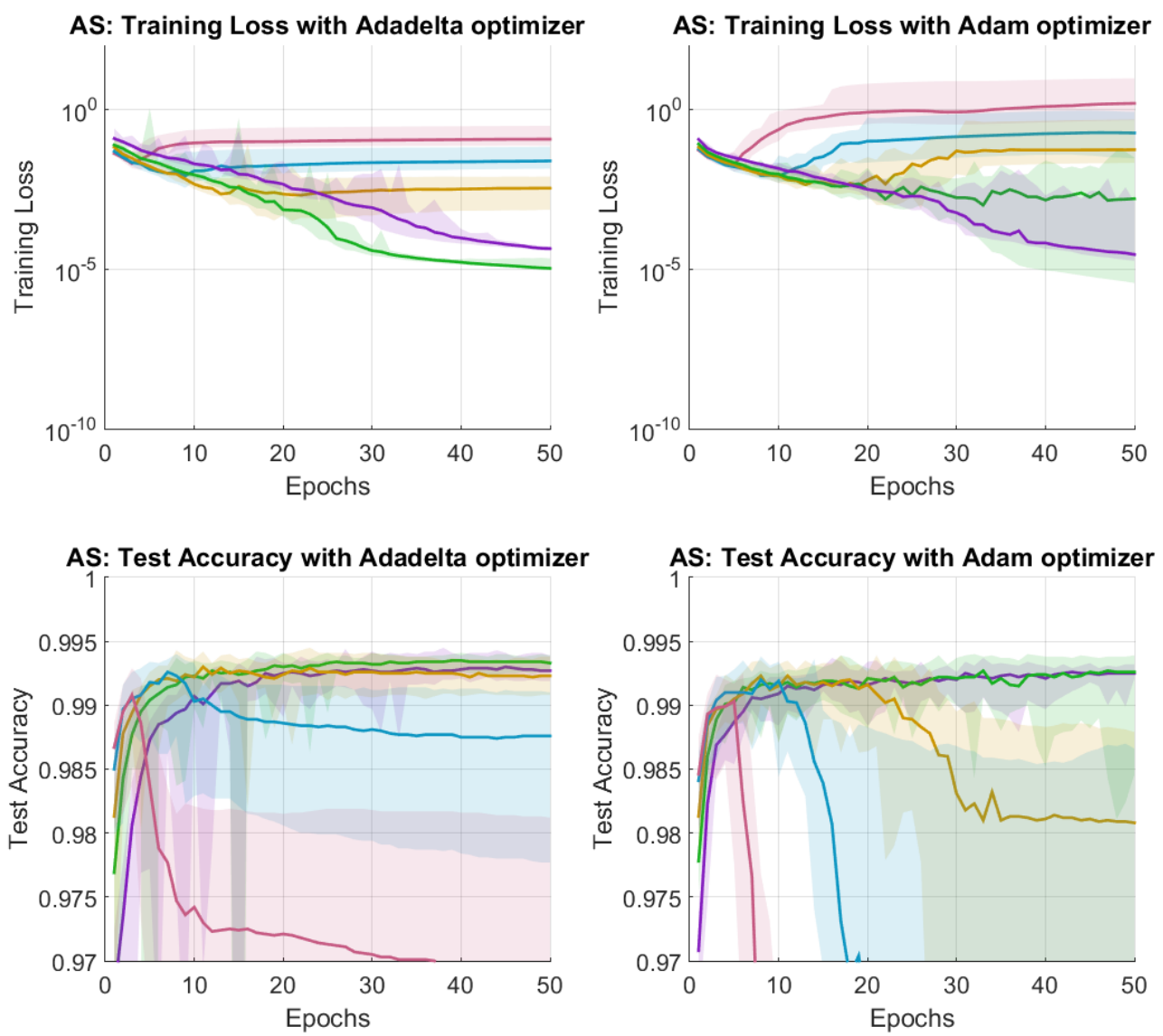

BS: $64-B S: 128-B S: 256-B S: 512-B S: 1024$

Fig. 1: Original Adaptive Sampling applied on the training of a CNN for the classification of the MNIST dataset. Each curve shows the median, and the shaded regions correspond to the min. and max. values over 15 trials. Colors illustrate different batch sizes (BS, bottom inset). Left column: AS with Adadelta; right column: AS with Adam.

The solution to (4) due to Gopal (2016) gives the optimal distribution $P$ at the $t$-th iteration:

$$
p_{j} \propto \frac{\left|c_{j}\right|}{N} \sqrt{\frac{1}{\left|c_{j}\right|} \sum_{i \in c_{j}}\left\|\nabla_{\theta} L\left(\varphi\left(x_{i}^{t} ; \theta^{t-1}\right), y_{i}^{t}\right)\right\|^{2}}
$$

In the work of Gopal (2016) AS was tested on three classification datasets using binary logistic regressors, and it was shown to effectively reduce the variance of the objective function employed. However, AS was not tested on the training of deep networks. Prior to the development of this work, we tested AS on the training of a CNN for classification of the MNIST dataset without obtaining satisfactory results (Fig. 1). This motivated further study that in turn led us to propose modifications to improve AS, which are described below.

\section{Regularized Adaptive Sampling}

As can be observed in Fig. 1, the loss function (plots on the top row) which should reach a value of approximately 10E-6 for the learning model to achieve good generalization (test accuracy above 0.995) is not sufficiently reduced by means of the original AS method. This leads to suboptimal generalization of the network (bottom row of Fig. 1). The lack of adequate convergence for different batch-sizes and different optimizers (as illustrated by the plots) led us to suspect that the cause was intrinsic to the AS algorithm, rather than the choice of hyper-parameters or optimizers employed.

Careful examination of the instances selected by AS shows that as training proceeds the probability of selecting instances from only one (or a very few) of the bins (i.e. classes) in the dataset increases dramatically (see top two rows in Fig. 2). As the network is shown examples from only one/a few of the problem classes, training fails and the generalization collapses; 

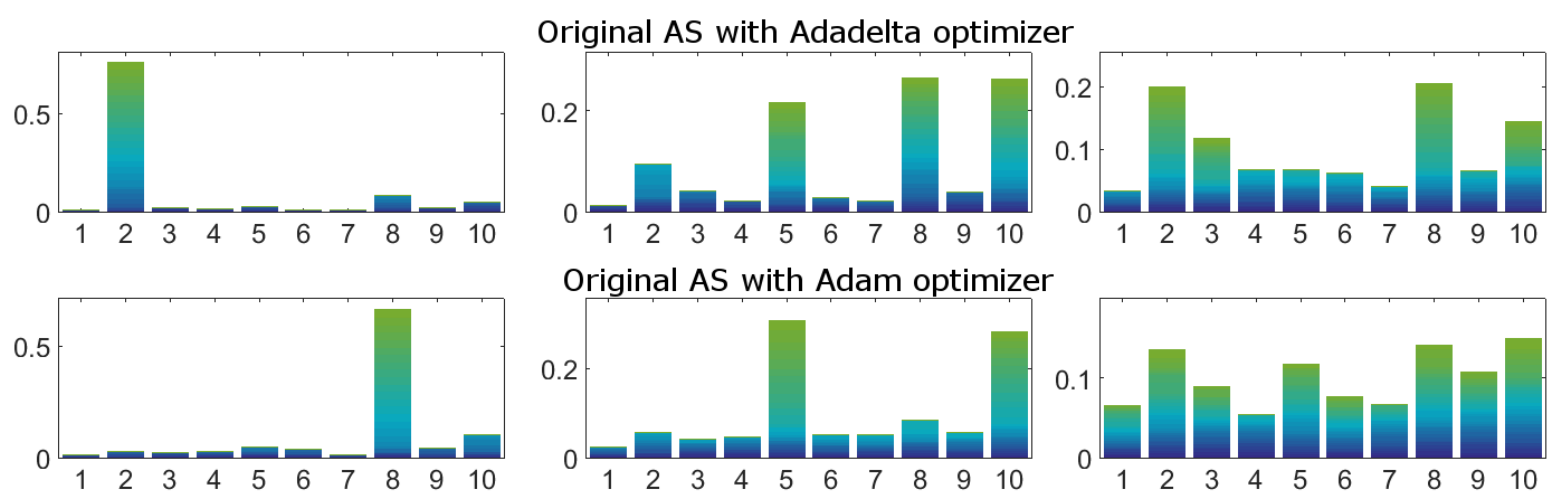

Modified AS (RAS) with Adadelta optimizer
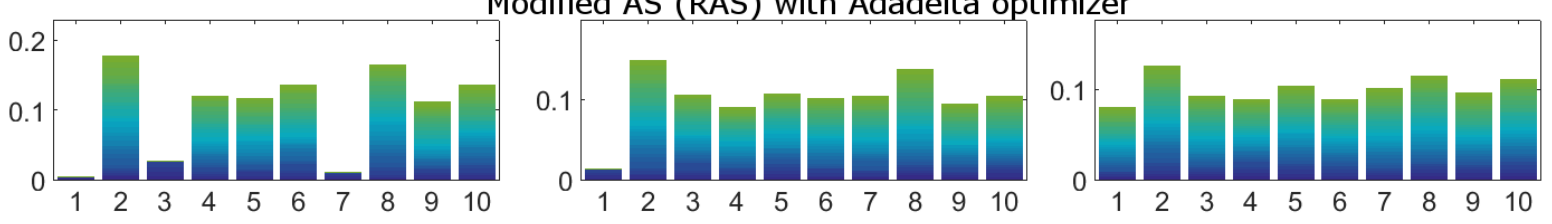

Modified AS (RAS) with Adam optimizer

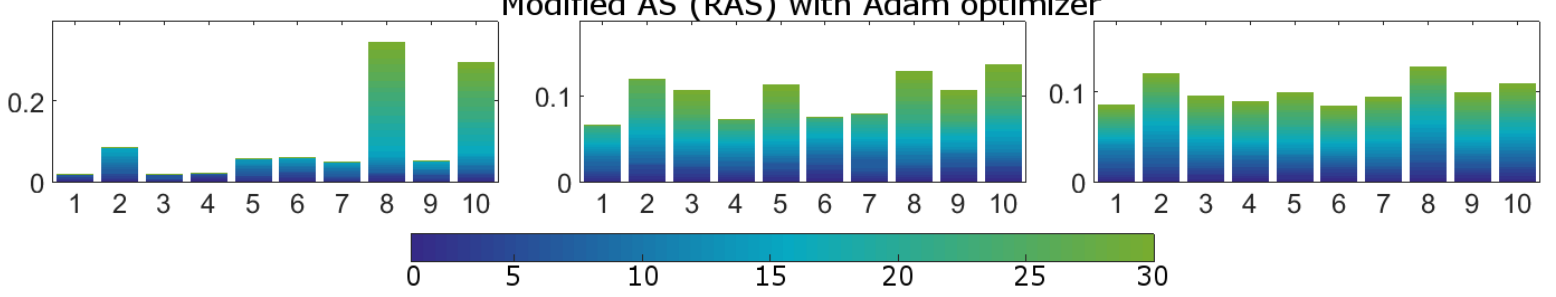

Fig. 2: Distribution of sampled MNIST instances over classes (1 to 10), along training epochs (according to bottom inset). Original AS with Adadelta ( $1^{\text {st }}$ row) and with Adam ( $2^{\text {nd }}$ row). Regularized AS with Adadelta ( $3^{\text {rd }}$ row) and with Adam ( $4^{\text {th }}$ row). Distributions for batch sizes 64,128 and 256, are shown in columns from left to right.

this is particularly notorious when small batches are employed, as the plots for batch-sizes 64 and 128 show. Although increasing the batch-size does help to maintain a more balanced selection over the bins, using large batch-sizes in general hinders the training process.

Derived from this observation, our insight is that the selection probability of any single bin in the partition of the dataset should not increase in excess relatively to the probabilities of the other bins, since this eventually causes the distribution to collapse onto a single bin. In other words, excessive growth of single bin's probabilities must be impeded with the aim of maintaining a balanced distribution of the sampled instances. We interpret this as a form of regularization over the bin probabilities and identify the modified AS method by the name of Regularized Adaptive Sampling (RAS). The regularization effect of the modifications introduced in RAS can be seen in the two bottom rows in Fig. 2 where, with the exception of the case for batch-size 64 with Adam optimizer, the distribution over the classes are perceptibly more balanced.
Examination of the instance-selection probability in AS shows that the second factor in (3), which represents the probability of the $i$-th instance to be selected within the $j$-th bin, is not connected to the loss value (or gradient of the loss w.r.t. the model's parameters) corresponding to that instance. In other words, the relative relevance of individual instances is lost in the computation of $P\left(x_{i}\right)$. To correct this, let us start by revisiting the motivation for IS.

Indeed, IS can be seen as a method to estimate (1), surrogating a uniform distribution with a non-uniform one. Thus we can pose a Monte Carlo (MC) estimator:

$$
\begin{aligned}
\theta^{*} & =\underset{\theta}{\arg \min } E_{x_{i} \sim \mathcal{V}}\left[\zeta\left(x_{i}\right) L\left(\varphi\left(x_{i} ; \theta\right), y_{i}\right)\right] \\
& =\underset{\theta}{\arg \min } \frac{1}{N} \sum_{i=1}^{N} \frac{1}{\mathcal{V}\left(x_{i}\right)} L\left(\varphi\left(x_{i} ; \theta\right), y_{i}\right)
\end{aligned}
$$

where $\zeta\left(x_{i}\right)=\mathcal{U}\left(x_{i}\right) / \mathcal{V}\left(x_{i}\right)$ is called the importance weight and $\mathcal{V}$ the importance distribution. Bouchard et al. (2015) showed that SGD is a powerful tool to optimize the sampling distribution of MC estimators, 
futher motivating the idea of employing a sampling distribution which is not fixed through time.

Our proposal is to incorporate a second, decidedly non-uniform probability distribution $Q^{j}$, defined over the elements of the $j$-th bin: $Q^{j}=\left\{q_{1}^{j}, q_{2}^{j}, \ldots, q_{\left|I_{j}\right|}^{j}\right\}$, where $q_{i}^{j}=\mathcal{P}\left(c_{j}=x_{i \in I_{j}}^{j}\right)$ and $I=\left\{I_{1}, I_{2}, \ldots, I_{k}\right\}$ are index sets of the partition $C$.

Assuming that $P$ and $Q^{j}$ are independent, the new probability of picking the $i$-th instance can be defined as $\mathcal{V}\left(x_{i}\right) \equiv \mathcal{P}\left(X=x_{i}\right)=p_{j} q_{i}^{j}$ so that $(7)$ becomes:

$$
\theta^{*}=\underset{\theta}{\arg \min } \frac{1}{N} \sum_{i=1}^{N} \frac{1}{p_{j} q_{i}^{j}} L\left(\varphi\left(x_{i} ; \theta\right), y_{i}\right) .
$$

Recalling that the main goal of using non-uniform sampling of the instances is to reduce the variance of the descent direction, the introduction of $Q^{j}$ generates a second problem similar to (4) but w.r.t. $Q^{j}$ :

$$
\min _{Q^{j}} V\left[d_{j}^{t}\right]=\min _{Q^{j}}\left(\mathbb{E}\left[d_{j}^{t}{ }^{\mathrm{T}} d_{j}^{t}\right]-\mathbb{E}\left[d_{j}^{t}\right]^{\mathrm{T}} \mathbb{E}\left[d_{j}^{t}\right]\right)
$$

Formally, the original gradient variance minimization problem is decomposed into two minimization problems, (4) and (9). Problem (4) is the minimization of the gradient variance w.r.t $P$ assuming a fixed $Q^{j}$ and (9) is the complementary minimization of the gradient variance w.r.t $Q^{j}$ assuming a fixed $P$.

The solution to (4) has already been given in (6) and can be simply re-written to accommodate $Q^{j}$ as:

$$
p_{j} \propto \frac{1}{N} \sqrt{\sum_{i \in c_{j}} \frac{1}{q_{i}^{j}}\left\|\nabla_{\theta} L\left(\varphi\left(x_{i}^{j} ; \theta^{t-1}\right), y_{i}^{j}\right)\right\|^{2}}
$$

For (9), use $1 / N=\left(\left|c_{j}\right| / N\right)\left(1 /\left|c_{j}\right|\right)$ in (8) so that:

$$
\begin{array}{r}
\frac{1}{N} \sum_{c_{j} \in C} \sum_{i \in c_{j}} \frac{1}{p_{j} q_{i}^{j}} L\left(\varphi\left(x_{i}^{j} ; \theta\right), y_{i}^{j}\right)= \\
\sum_{c_{j} \in C} \frac{1}{p_{j}} \frac{\left|c_{j}\right|}{N} \frac{1}{\left|c_{j}\right|} \sum_{i \in c_{j}} \frac{1}{q_{i}^{j}} L\left(\varphi\left(x_{i}^{j} ; \theta\right), y_{i}^{j}\right)
\end{array}
$$

and notice that the last summation can be separated to pose the following optimization problem exclusively over the instances of the $j$-th bin $\forall j=1, \ldots, k$ :

$\theta_{q}^{*}=\underset{\theta}{\arg \min } \frac{1}{\left|c_{j}\right|} \sum_{i \in c_{j}} \frac{1}{q_{i}^{j}} L\left(\varphi\left(x_{i}^{j} ; \theta\right), y_{i}^{j}\right)$,

so that the gradient $d_{j}^{t}$ to be used in (9) is:

$$
d_{j}^{t}=\frac{1}{q_{i}^{j}} \frac{1}{\left|c_{j}\right|} \nabla_{\theta} L\left(\varphi\left(x_{i}^{j} ; \theta^{t-1}\right), y_{i}^{j}\right) .
$$

It can be shown (cf. Avalos-López et al. (2021)) that $\mathbb{E}\left[d_{j}^{t}\right]$ is independent of $q_{i}^{j}$ and can be ignored in the solution of (9). We are left with $\mathbb{E}\left[d_{j}^{t \mathrm{~T}} d_{j}^{t}\right]$ and proceed to minimize the variance following (Gopal (2016)). Finally, the optimal $Q^{j}$ at the $t$-th iteration is given by:

$$
q_{i}^{j} \propto \frac{1}{\left|c_{j}\right|}\left\|\nabla_{\theta} L\left(\varphi\left(x_{i}^{j} ; \theta^{t-1}\right), y_{i}^{j}\right)\right\| .
$$

Intuitively, the selective sampling implemented by RAS can be understood as two consecutive selection procedures: first a bin is selected according to (10) and then training instances within that bin are selected according to (14). It is worth remarking that in both cases the derived distributions are directly proportional to the norm of $\nabla_{\theta} L$ since this optimizes the criterion of the minimum variance of the descent direction, which is known to improve training convergence. The pseudocode of our RAS proposal for training of deep networks is given in Algorithm 1.

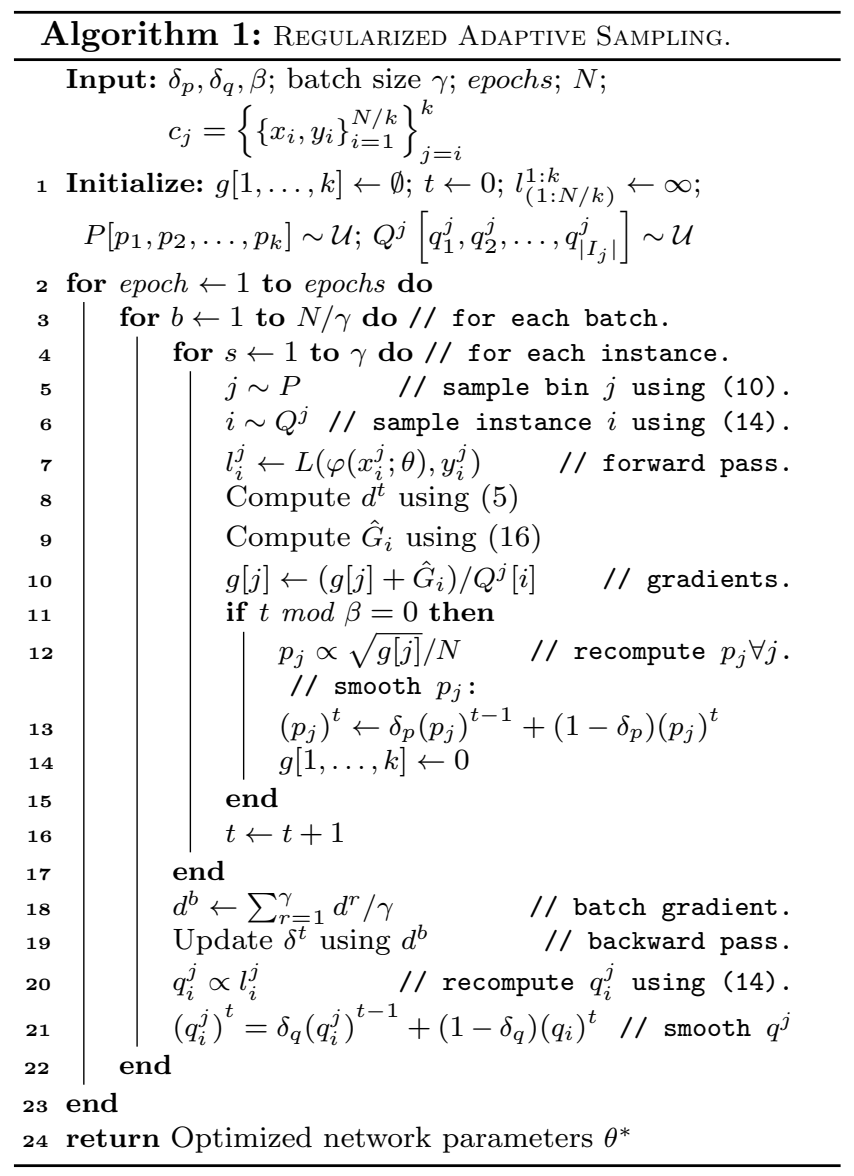




\subsection{Computational considerations}

In the implementation of the RAS method there are a few computational drawbacks that must be addressed:

1. It is impractical to reset the sampling distribution (10) after each iteration. Instead, $p_{j}$ can be updated every $\beta$ iterations (see line 11 in Algorithm 1).

2. Computing the gradient norm for an individual instance requires computing a square root. However, Katharopoulos and Fleuret (2017) show that sampling using the loss (which is readily available during training) exhibits similar variance reducing properties to sampling according to the gradient norm. Thus, (14) can be replaced with:

$$
q_{i}^{j} \propto L\left(\varphi\left(x_{i}^{j} ; \theta\right), y_{i}^{j}\right)
$$

3. During training, $P$ and $Q^{j}$ may degenerate to zero for some bins or some instances, respectively, causing those bins/instances to never be selected again. To avoid this, $P$ and $Q^{j}$ are smoothed to mitigate the appearance of extreme values. This requires two more hyper-parameters, $\delta_{p}, \delta_{q} \in(0,1)$, to modulate the smoothing of $P$ and $Q^{j}$, respectively.

4. Computing the full gradient becomes prohibitive for deep learning models with millions of parameters. Katharopoulos and Fleuret (2018) derived an upperbound of the gradient norm:

$$
\begin{aligned}
\hat{G}_{i} & \equiv\left\|\nabla_{\theta_{t}^{l}} L\left(\varphi\left(x_{i}^{j} ; \theta^{t-1}\right), y_{i}^{j}\right)\right\|^{2} \\
& \geq\left\|\nabla_{\theta} L\left(\varphi\left(x_{i}^{j} ; \theta^{t-1}\right), y_{i}^{j}\right)\right\|^{2}
\end{aligned}
$$

that can be employed in lieu of the full gradient. In (16) $\nabla_{\theta_{t}^{l}} L\left(\varphi\left(x_{i}^{j} ; \theta^{t-1}\right), y_{i}^{j}\right)$ is the gradient w.r.t. the pre-activation outputs of the last layer in a DNN.

\section{Experimental Setup and Results}

The proposed RAS technique is compared against uniform sampling (i.e. typical SGD) and against a lossrank-based selection strategy, OBS (Loshchilov and Hutter (2015)). This comparison is carried out using two well-known benchmark classification problems contained in the MNIST dataset (LeCun et al. (1998)) and the CIFAR-10 dataset (Krizhevsky et al. (2009)).

The standard MNIST dataset is a very popular benchmark for evaluation of classification models because it can be easily solved by small modern networks. For this dataset a CNN with two convolutional layers (each Batch Normalized and followed by a Max-pooling layer) and two fully connected (FC) layers was implemented (see Table 1). The ReLu activation function was used for all the layers except for the last one, which employs the Softmax cross-entropy loss as is commonly done for classification problems. Dropout (with a rate of 0.5 ) and data normalization was used on the first FC layer.

For the CIFAR-10 dataset, which is a more difficult problem, a standard VGG-16 CNN (Simonyan and Zisserman (2015)) was implemented. Batch normalization (for each convolutional layer) and Dropout (for the FC layers) were employed. All the layers included the ReLu activation function, except the output layer, wich used the Softmax cross-entropy loss. The architecture

\begin{tabular}{|c|c|c|}
\hline $\begin{array}{l}\text { Layer } \\
\text { Type }\end{array}$ & $\begin{array}{c}\text { Depth } \\
\text { (in : out) }\end{array}$ & $\begin{array}{c}\text { Activation } \\
\text { Function }\end{array}$ \\
\hline Input $(28 \times 28$ image $)$ & 1 channel & - \\
\hline Conv. $(5 \times 5)+$ Batch Norm. & $1: 32$ & ReLU \\
\hline Max. Pooling $(2 \times 2)$ & $32: 32$ & - \\
\hline Conv. $(5 \times 5)+$ Batch Norm. & $32: 32$ & ReLU \\
\hline Max. Pooling $(2 \times 2)$ & $32: 32$ & - \\
\hline Flattening & $32: 512$ & - \\
\hline $\mathrm{FC}$ & 256 units & ReLU \\
\hline $\mathrm{FC}$ & 10 units & SoftMax \\
\hline
\end{tabular}
is provided in Table 2 .

Table 1: CNN Architecture for MNIST classification

Table 2: CNN architecture (VGG-16) for CIFAR-10

\begin{tabular}{ccc} 
Layer & Depth & Activation \\
Type & (in : out) & Function \\
\hline Input $(32 \times 32$ image $)$ & 3 channels & - \\
Conv. $(3 \times 3)+$ Batch Norm. & $3: 64$ & ReLU \\
Conv. $(3 \times 3)+$ Batch Norm. & $64: 64$ & ReLU \\
Max. Pooling $(2 \times 2)$ Stride: & $64: 64$ & - \\
Conv. $(3 \times 3)+$ Batch Norm. & $64: 128$ & ReLU \\
Conv. $(3 \times 3)+$ Batch Norm. & $128: 128$ & ReLU \\
Max. Pooling $(2 \times 2)$ Stride:2 & $128: 128$ & - \\
Conv. $(3 \times 3)+$ Batch Norm. & $128: 256$ & ReLU \\
Conv. $(3 \times 3)+$ Batch Norm. & $256: 256$ & ReLU \\
Max. Pooling $(2 \times 2)$ Stride:2 & $256: 256$ & - \\
Conv. $(3 \times 3)+$ Batch Norm. & $256: 512$ & ReLU \\
$2 \times$ Conv. $(3 \times 3)+$ Batch Norm. & $512: 512$ & ReLU \\
Max. Pooling $(2 \times 2)$ Stride:2 & $512: 512$ & - \\
$3 \times$ Conv. $(3 \times 3)+$ Batch Norm. & $512: 512$ & ReLU \\
Max. Pooling $(2 \times 2)$ Stride:2 & $512: 512$ & - \\
Flattening & $512: 4096$ & - \\
FC & 4096 units & ReLU \\
FC & 4096 units & ReLU \\
FC & 10 units & SoftMax \\
\hline
\end{tabular}

Both of these datasets correspond to classification problems with ten classes and present a similar structure and size. The MNIST dataset contains 60,000 training instances and 10,000 test instances, all of which are gray-scale images of $28 \times 28$ pixels representing handwritten digits. The CIFAR-10 dataset includes 50,000 

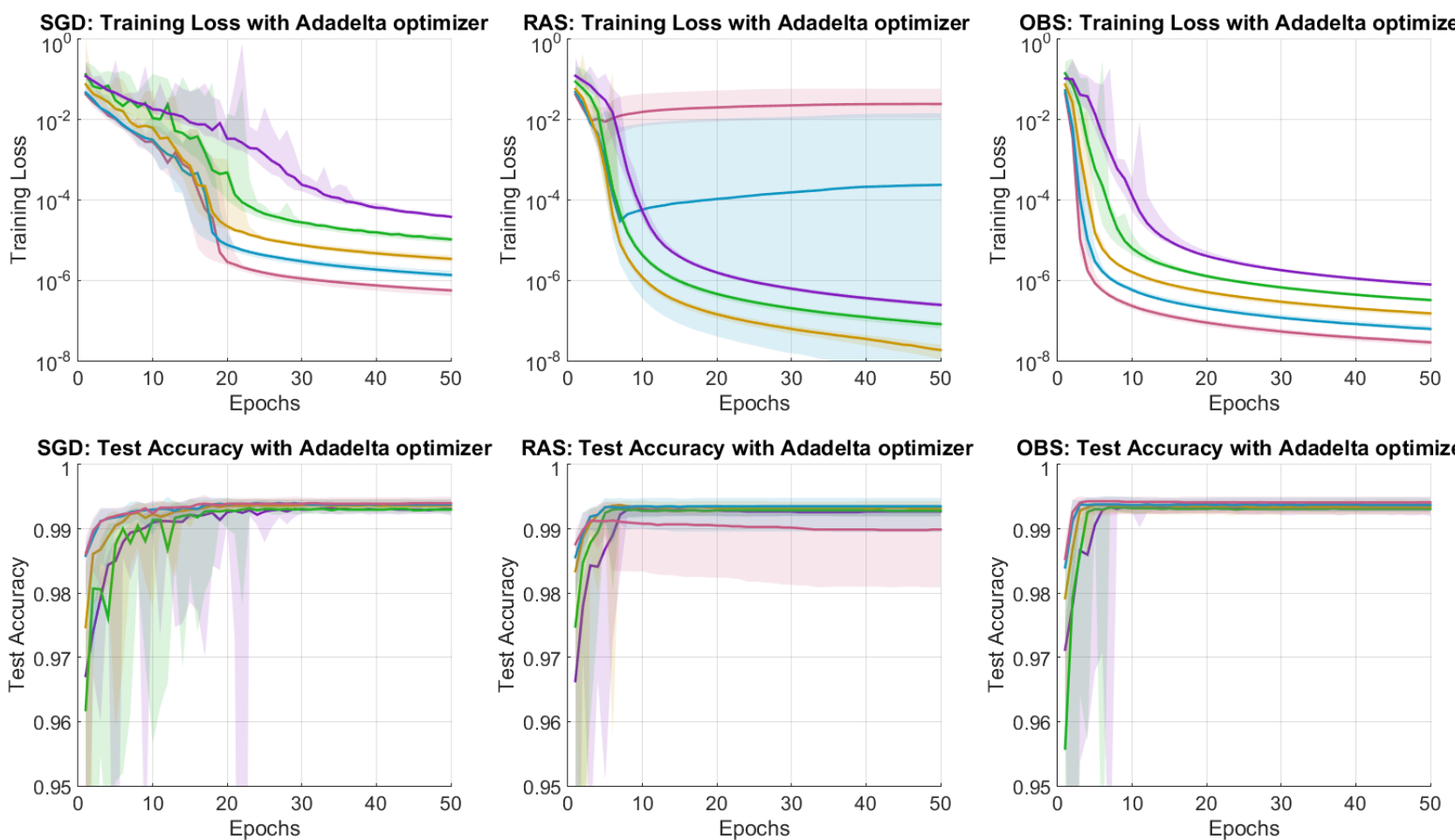

OBS: Test Accuracy with Adadelta optimizer

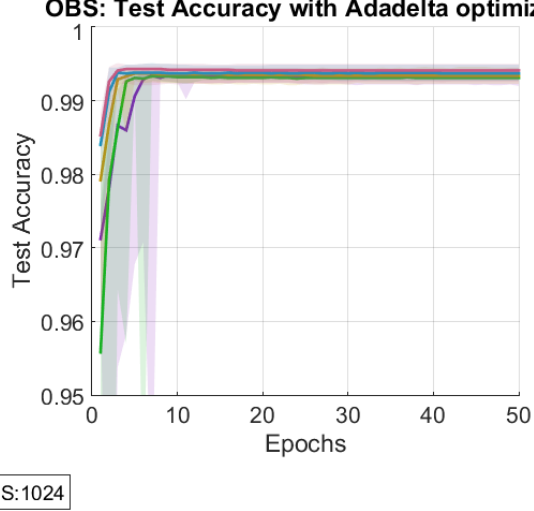

Fig. 3: Comparison of RAS vs SGD and OBS for classification of MNIST. Top row: Training loss. Bottom row: corresponding test accuracy. Median (solid curve), min. and max. values (shaded region) over 15 trials for different batch sizes (see bottom inset). All compared techniques were combined with the Adadelta optimizer.

training instances and 10,000 test instances, as color images of $32 \times 32$ pixels that represent different classes of vehicles and animals. The original instances were used without data-augmentation and without validation subsets (i.e. the datasets are initially split into training and test subsets without overlap and this split is maintained throughout the experiments).

Typical base-line classification performance on the MNIST dataset is above 99\%, while a good base-line performance of VGG-16 on the CIFAR-10 dataset could be set around $85 \%$ correct classification (Kawaguchi et al. (2020), Wang (2020) $)^{2}$. Notice that in our comparison we are not interested in obtaining top-level performance of the networks, but rather, in the characterization of the compared sampling methods under a range of experimental conditions such as different problems, optimizers, and a range of batch sizes.

All of the compared methods were implemented using the library PyTorch ${ }^{3}$ for Python 3.6 and the experiments were executed on a Linux box (with Ubuntu

2 This base-line is harder to establish because of the problem complexity, the hyper-parameters and advanced techniques that can be used, but in the literature we encountered similar experiments with results around this value.

3 https://pytorch.org/
Bionic Beaver OS) equipped with a GeForce GTX 1070 GPU. The experiments consisted in training each of the CNNs for a fixed number of epochs (50 for MNIST and 40 for CIFAR-10 since the VGG-16 network is much more expensive to run) and recording the training-loss and test-accuracy per epoch. For all the experiments, the side-information in RAS was set to be the classlabels, and the hyper-parameters were set as follows: $\beta=3, \delta_{p}=0.9$ and $\delta_{q}=0.1$; these $\delta$ values were selected through previous experimentation. OBS requires two extra hyper-parameters whose tried-out values can be found in (Loshchilov and Hutter (2015)) and based on those we set: $r=0.5$ and $s=10^{8}$.

In general (as discussed in Section 1), training of a deep network through pure SGD can be inefficient; because of this, training of networks for practical use is usually carried out with the help of improved training algorithms known as optimizers. In this work the compared instance-selection methods are combined with two of the most popular optimizers (with their default parameters in Pytorch $\left.^{4}\right)$ : Adadelta $(\mathrm{l}=1.0, \mathrm{rho}=0.9$, eps $=1 \mathrm{e}-06$, weight_decay $=0)$ and Adam $(\mathrm{lr}=0.001$, betas $=(0.9,0.999)$, eps $=1 \mathrm{e}-08$, weight_decay $=0$, amsgrad

\footnotetext{
4 https://pytorch.org/docs/stable/optim.html\#algorithms
} 

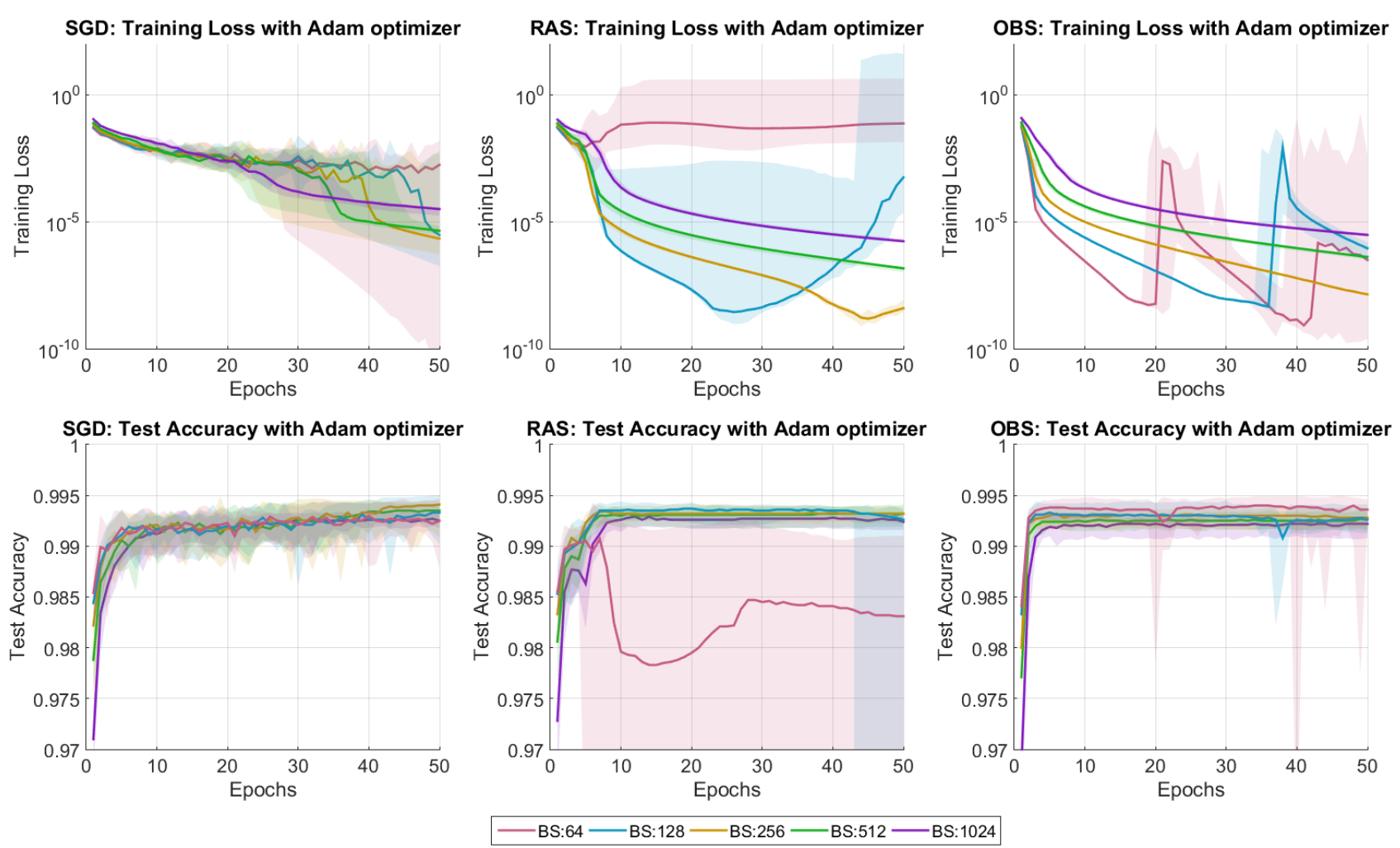

Fig. 4: Comparison of RAS vs SGD and OBS for classification of MNIST. Top row: Training loss. Bottom row: corresponding test accuracy. Median (solid curve), min. and max. values (shaded region) over 15 trials for different batch sizes (BS, see bottom inset). All compared techniques were combined with the Adam optimizer.

$=$ False). Also, the use of mini-batches in SGD-training of DNNs is practically universal, although, regardless of its significant effect, the size of the batches to be used is a free parameter that needs to be empirically set by the user. Usually, when selecting a batch-size there is a tradeoff between speed and performance; smaller batchsizes imply more training time required but can generate better performance of the trained networks, while larger batch-sizes require shorter training time but the obtained networks may not perform as well. With the objective of obtaining a characterization as complete as possible, all of the experiments consider batch-sizes from $2^{6}=64$ to $2^{10}=1024$ instances.

The first set of results, for MNIST with Adadelta, is shown in Fig. 3. Notice that although the training loss with RAS does not converge for batch-sizes of 64 and 128 instances, in the other three cases the training with RAS is faster (in terms of epochs, elapsed times are discussed below) than with SGD and OBS. The second set of results, for MNIST with Adam optimizer, is shown in Fig. 4. In this case it can be observed that convergence is very challenging for SGD, while OBS and RAS have some difficulties with batch-sizes 64 and 128 but in general show better convergence than SGD.
The third set of results, for CIFAR-10 with Adadelta, is presented in Fig. 5. In this figure it can be seen that, with the exception of batch-size 1024 and the last 10 epochs of batch-size 64, the training-loss and the test-accuracy plots of RAS are in general more stable (i.e. show smaller dispersion and fluctuation) than those of OBS and SGD. In the Fourth set of results, for CIFAR-10 with Adam, shown in Fig. 6, the training-loss and test-accuracy plots of SGD and RAS are relatively smooth and very similar between methods, while the plots of OBS show that this method fails to converge for batch-sizes of 256, 512 and 1024 instances.

Finally, the average training times per epoch required by each combination of a sampling method and an optimizer are shown in Fig. 7 as plots for different batch-sizes of the networks trained for MNIST and CIFAR-10. Note that the times required for training on CIFAR-10 are about an order of magnitude larger than those required for training on MNIST. Also, that as the batch-size increases the training time decreases (in accordance with the literature (Smith et al. (2017))), and that the difference in required time between methods is quite significant for the simple problem of MNIST but not so for the more complex problem of CIFAR-10. 


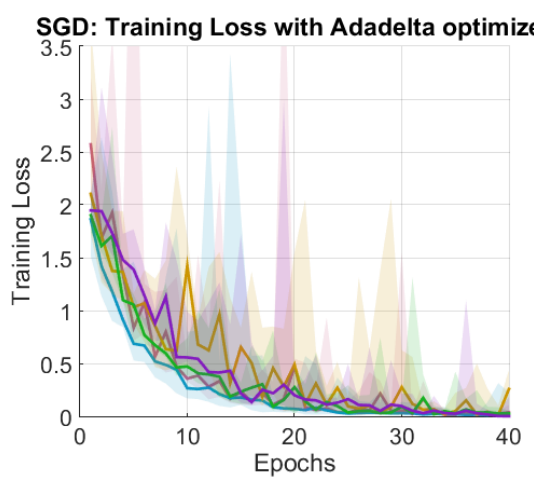

SGD: Test Accuracy with Adadelta optimize

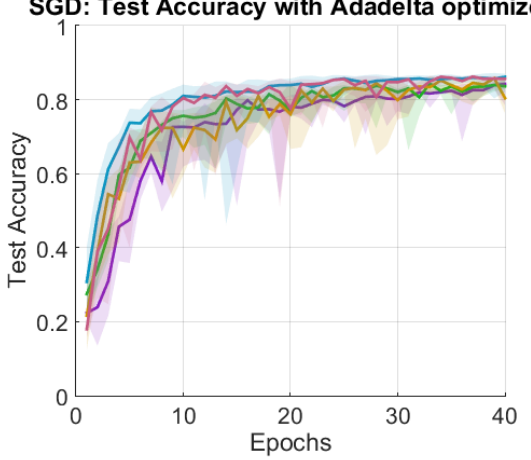

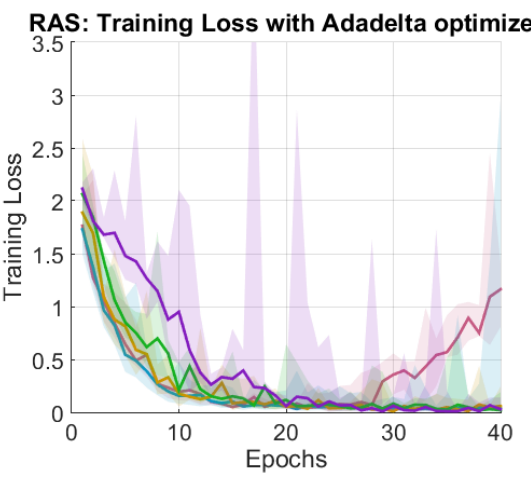

RAS: Test Accuracy with Adadelta optimizer

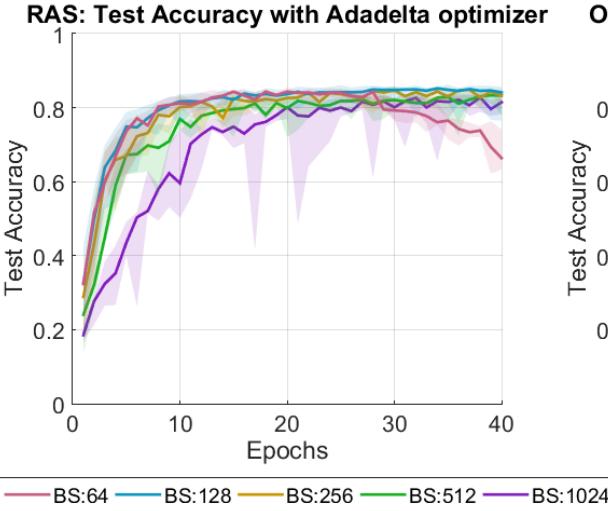

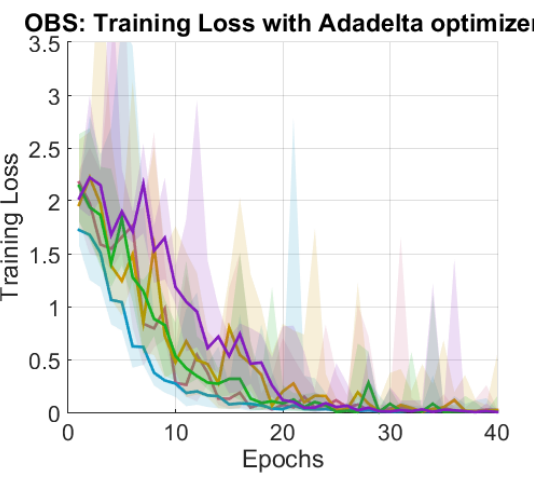

OBS: Test Accuracy with Adadelta optimizer

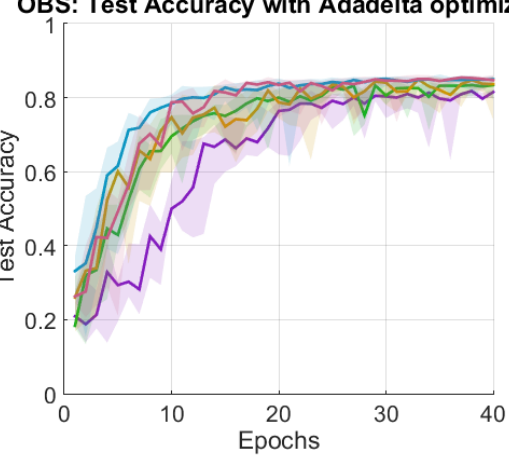

Fig. 5: Comparison of RAS vs SGD and OBS for classification of the CIFAR10 dataset. Top row: Training loss. Bottom row: corresponding test accuracy. Median (solid curve), min. and max. values (shaded region) over five trials for different batch sizes (BS, bottom inset). All compared techniques were combined with the Adadelta optimizer.

\section{Discussion}

Considering our set of results as a whole, it can be appreciated that there is a wide variety of individual behaviors of the compared methods that can be analyzed in terms of the convergence of the loss function. These can be broadly separated into two groups: i) Experiments where the methods behave as expected (success) and ii) Experiments where the methods misbehave $^{5}$ (failure). For instance, on the MNIST dataset with Adadelta optimizer (Fig. 3), RAS failed in the experiments for batch-size 64 and 128, but succeeded in the rest of the experiments. For the same dataset and optimizer, SGD and OBS always behaved as expected.

Table 3 provides a visual summary of the qualitative outcome of the compared methods over all the experiments, where a success is represented by a green circle $(\bullet)$ and a failure by a red square ( $\square$ ). The bottom row shows the success rate per column and the rightmost column the success rate per experimental setup, which

5 Slow convergence as well as large oscillations of the loss function are considered inconsistent with the desired behavior, even if the final test accuracy is good. In other words, the whole training process is examined, not just the end result. are described as the combination of a dataset and an optimizer: 1.-MNIST w/Adadelta; 2.-MNIST w/Adam; 3.-CIFAR-10 w/Adadelta; 4.-CIFAR-10 w/Adam.

Table 3: Summary of qualitative results

\begin{tabular}{|c|c|c|c|c|c|c|c|}
\hline & Setup & 64 & 128 & 256 & 512 & 1024 & \\
\hline \multirow{4}{*}{$\bigoplus_{\mho}$} & 1 & 0 & 0 & 0 & 0 & 0 & 1 \\
\hline & 2 & $\square$ & $\square$ & $\square$ & $\square$ & $\square$ & 0 \\
\hline & 3 & 0 & 0 & $\square$ & 0 & $\square$ & 0.6 \\
\hline & 4 & 0 & 0 & 0 & 0 & 0 & 1 \\
\hline \multirow{4}{*}{$\underset{\sim}{\stackrel{\pi}{L}}$} & 1 & $\square$ & 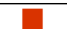 & 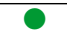 & 0 & 0 & 0.6 \\
\hline & 2 & $\square$ & $\mathbf{\square}$ & 0 & 0 & ○ & 0.6 \\
\hline & 3 & $\square$ & 0 & 0 & 0 & $\square$ & 0.6 \\
\hline & 4 & 0 & 0 & 0 & 0 & 口 & 0.8 \\
\hline \multirow{5}{*}{$\stackrel{\text { กी }}{0}$} & 1 & 0 & 0 & O & 0 & 0 & 1 \\
\hline & 2 & $\square$ & 口 & 0 & 0 & 0 & 0.6 \\
\hline & 3 & $\square$ & 0 & 口 & 口 & 口 & 0.2 \\
\hline & 4 & 0 & 0 & 口 & 口 & $\square$ & 0.4 \\
\hline & & 0.5 & 0.66 & 0.66 & 0.75 & 0.5 & O \\
\hline
\end{tabular}

The average success rate per method is SGD: 0.65, RAS: 0.65 and OBS: 0.55 . As recorded in Table 3, employing extreme batch sizes results in lower success rates overall. Focusing on the central values of the batch size (256 and 512) it can be seen that only the RAS method 

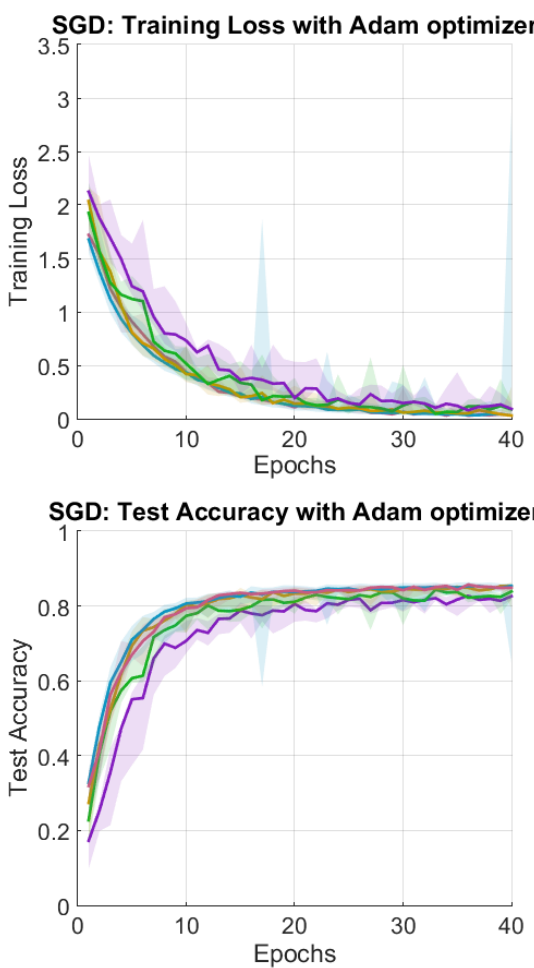

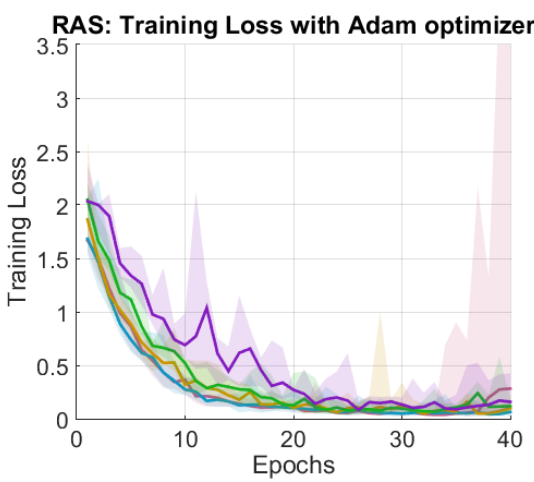

RAS: Test Accuracy with Adam optimizer

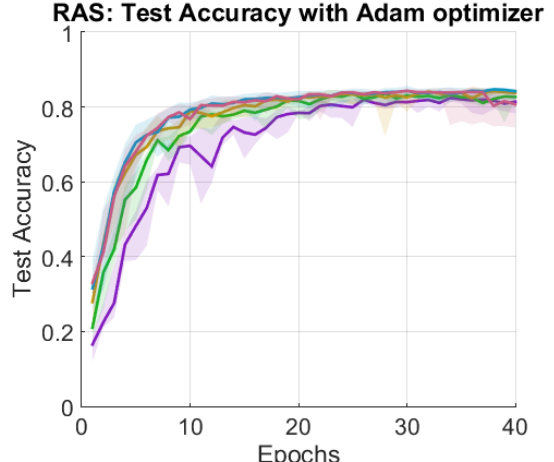

$-\mathrm{BS}: 64-\mathrm{BS}: 128-\mathrm{BS}: 256-\mathrm{BS}: 512-\mathrm{BS}: 1024$

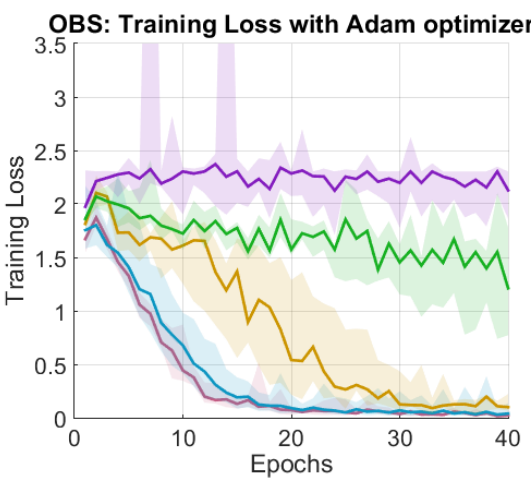

OBS: Test Accuracy with Adam optimizer

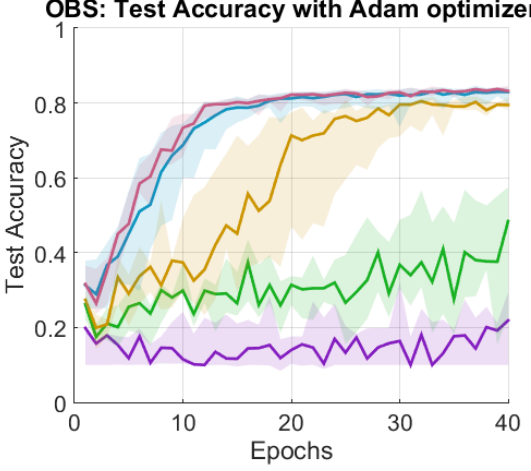

Fig. 6: Comparison of RAS vs SGD and OBS for classification of the CIFAR10 dataset. Top row: Training loss. Bottom row: corresponding test accuracy. Median (solid curve), min. and max. values (shaded region) over five trials for different batch sizes (BS, bottom inset). All compared techniques were combined with the Adam optimizer.

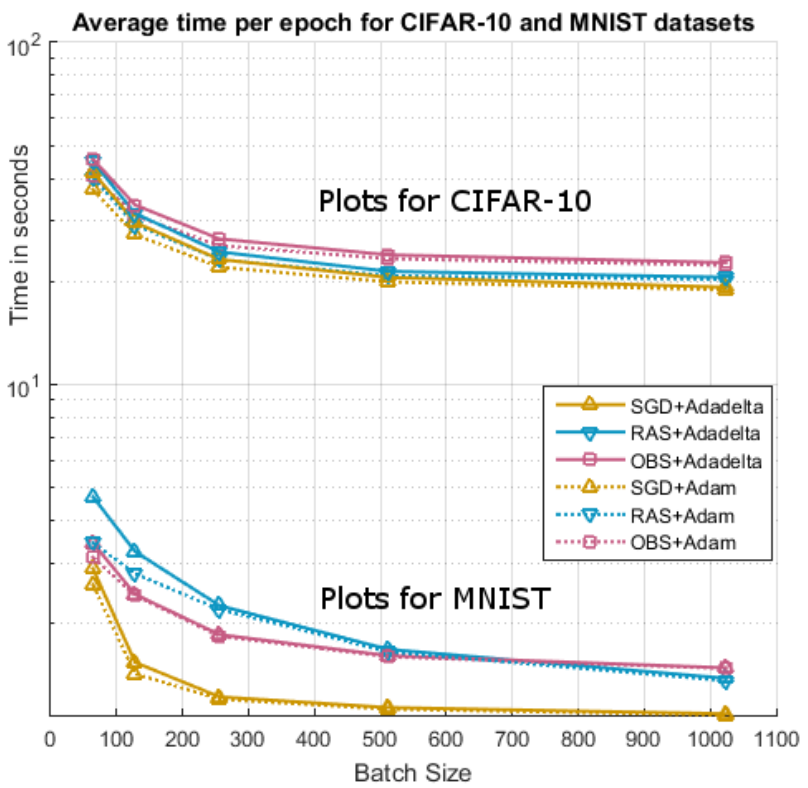

Fig. 7: Average training time per epoch. Times required for the CIFAR-10 dataset are about an order of magnitude larger than those required for the MNIST dataset. is $100 \%$ successful along the four experimental setups. The success of SGD is $62.5 \%$ and OBS is successful in only $50 \%$ of the experiments with these batch-sizes.

Why is it that the methods misbehave if one chooses the smallest or largest batch-sizes? For excessively small batches, a possible explanation in the case of the selective sampling methods is that small amounts of instances do not allow the computation of reliable estimates of the required probabilities; however, loss plots that increase over time instead of decreasing (see RAS in Fig. 3 and Fig. 4), or that show abrupt and significant jumps (see OBS in Fig. 4) can also be indicative of excessively large learning rates. On the other hand, the effect of very large batch sizes is more easily understood and expected. An excessive amount of instances in a batch counteracts the selectivity of the algorithms, this increases the variance of the estimates and slows down the convergence (in terms of loss reduction per epoch) or even prevents it entirely.

Based on the results described above, it is considered that the smaller batch-sizes (64 and 128) sometimes generate quite significant difficulties to some methods (and may make them more sensitive to the hyperparameters), while the larger batch-sizes (512 and par- 


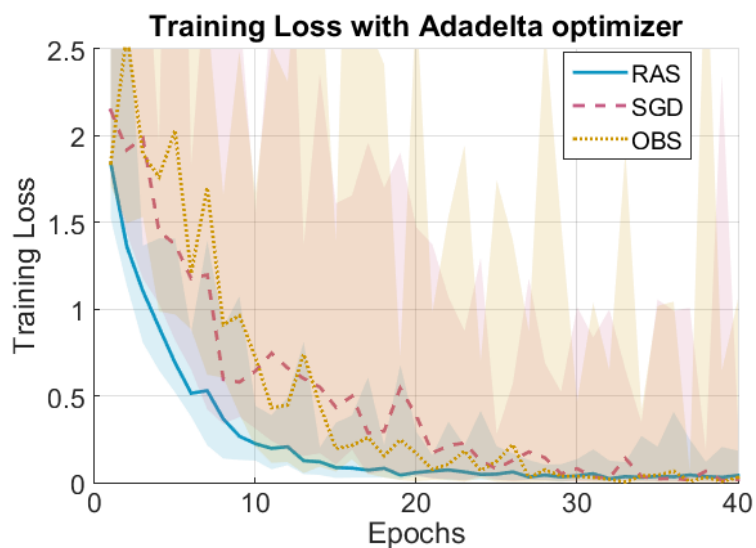

Test Accuracy with Adadelta optimizer

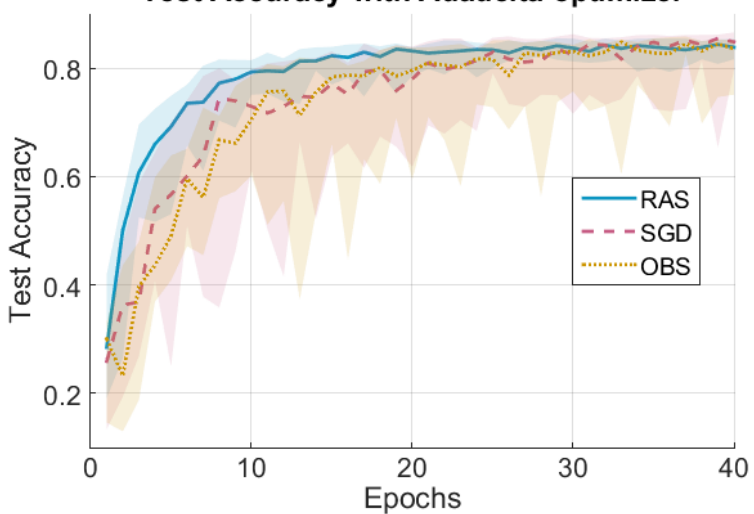

Fig. 8: Comparison between RAS, SGD and OBS for CIFAR-10 with Adadelta and batch-size: 256.

ticularly 1024) almost always generate inferior results for all methods. Because of this, and to simplify further analysis, a batch size of 256 (located in the middle of the range of batch-sizes considered) is regarded as a convenient compromise for all three methods.

The results for batch-size: 256 are shown in Fig. 8 (with Adadelta) and Fig. 9 (with Adam); these are limited to the CIFAR-10 dataset because it is a problem of more complexity than MNIST. The plots show the median and range over 15 trials. Regarding Fig. 8 it can be observed that the training loss curve obtained by RAS is smoother and decreases faster than the curves of SGD and OBS. The Loss of OBS intersects that of RAS at epoch 27 and the one of SGD does so until epoch 31. This is mirrored by the Test Accuracy, for which OBS equals RAS at epoch 33 and SGD does so at epoch 31. On average, there is an advantage of 30.5 epochs in favor of RAS. Furthermore, the dispersion of the data (illustrated by the shaded region around each curve) is much larger for SGD and for OBS than for RAS. This observation applies to both the Training Loss as well as to the Test Accuracy. In brief, it can be concluded that in this test it is preferable to choose RAS.

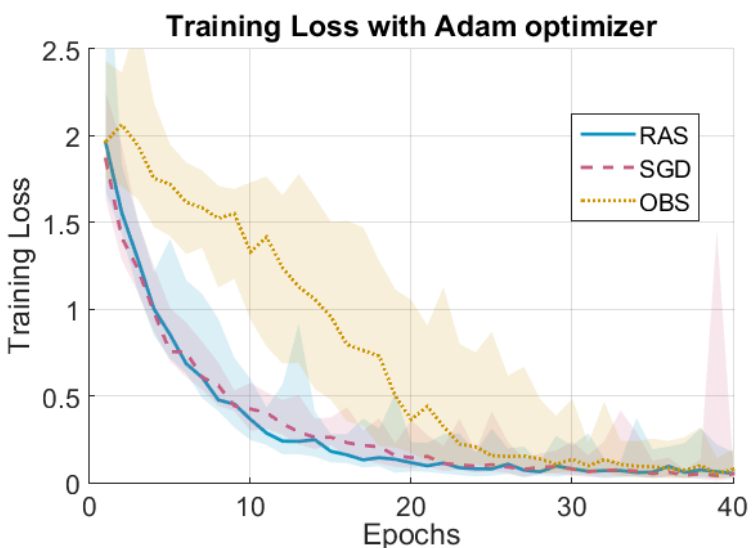

Test Accuracy with Adam optimizer

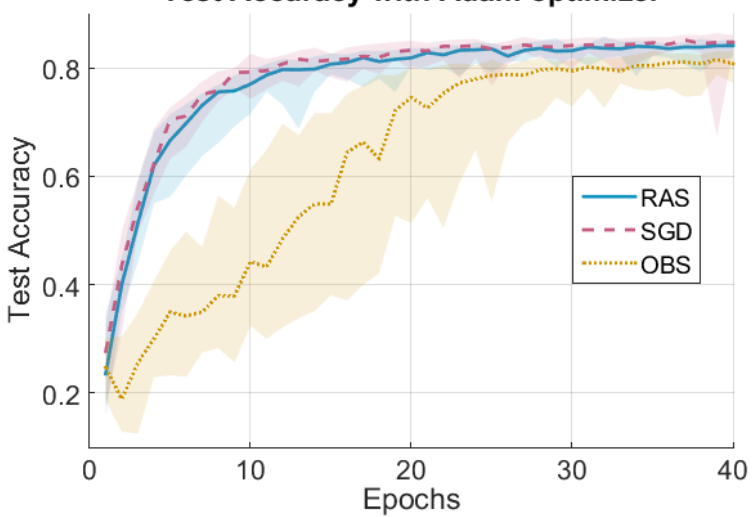

Fig. 9: Comparison between RAS, SGD and OBS for CIFAR-10 with Adam and batch-size: 256

The results in Fig. 9 show a different scenario to that described above. According to these, the loss and accuracy obtained by RAS are essentially equivalent to those of SGD (in median value as well as dispersion) throughout the whole training process. In contrast, the loss curve of OBS describes quite a different path and only intersects that of RAS near the end of the training, at epoch 36 out of 40 . This is reflected in the test accuracy curves, for which the curve of OBS never reaches the accuracy level obtained by RAS and SGD.

Finally, the differences between the median (over 15 trials) of the highest test accuracy obtained by RAS and the other methods are, using Adadelta: RAS-OBS= $+0.09 \%$; RAS-SGD $=-0.63 \%$, and using Adam: RAS$\mathrm{OBS}=+2.84 \%$; RAS-SGD $=-0.84 \%$. As can be seen, there are negligible differences (smaller than 1\%) between the test accuracy that RAS can obtain and that of the other methods. The only exception is the difference between RAS and OBS using Adam, which is of $2.84 \%$ in favor of RAS. From this, it can be said that while RAS sometimes offers the benefit of a more stable and faster convergence it does so without negatively affecting the final performance of the trained networks. 


\section{Conclusion}

A novel method to sample data in order to form mini batches for the training of DNNs has been described. This method is an improved IS-based adaptive sampling strategy designed to accelerate the training process, which works by defining optimal distributions over data bins (i.e. problem classes) and instances that reduce the variance of the gradient-norms, and lead to faster and smoother convergence of a network's parameters. Based on a experimental comparison including two classification problems of different complexity, two popular optimizers and a range of batch-sizes, it was shown that the proposed RAS method either matches or outperforms standard SGD and always surpasses the performance of another selective sampling method (OBS).

It was also shown that while different optimizers cause SGD and OBS to behave quite differently, our proposed method is more robust to this variable. However, as the other methods compared, RAS is sensitive to the choice of the batch-size employed. All of the remaining hyper-parameters involved were kept fixed to simplify the analysis. Although the RAS method implies a computational overhead that results in longer elapsed real times per epoch, this is only significant in relation to the very short times required for the training of the simple MNIST problem.

Variations of the proposed sampling strategy can be easily implemented, such as switching on/off the selective sampling at times when a speed-up is guaranteed, an idea presented by Katharopoulos and Fleuret (2018). Also, there is a variety of choices for the side information employed to define the importance of the bins and instances. For instance, Joseph et al. (2019) have explored the use of the entropy of the model as a measure of how informative are the instances in a training iteration. These ideas are being considered for future work.

Author contributions A.R.D. conceived of the study, participated in the design of the algorithms and implementation of the models; carried out computational experiments, analyzed experimental results, and wrote the paper with the other authors. M.O.R. participated in the design of the study and initial versions of the manuscript. M.C. participated in the design of the algorithms and analyzed the results. S.I.V. participated throughout in the preparation of the paper. All authors read and approved the final manuscript.

Funding This work was partially supported by The National Council of Science and Technology of Mexico (CONACYT) through grants: CÁTEDRAS-2598 (A. Rojas) and CÁTEDRAS-7795 (S.I. Valdez).
Availability of data and material Data are available from the authors upon reasonable request.

\section{Declarations}

Competing interest The authors declare that they have no competing interests.

Ethics approval and consent to participate Not Applicable.

Consent for publication Not Applicable.

\section{References}

Alain G, Lamb A, Sankar C, Courville A, Bengio Y (2015) Variance reduction in sgd by distributed importance sampling. arXiv preprint arXiv:151106481

Alom MZ, Taha TM, Yakopcic C, Westberg S, Sidike P, Nasrin MS, Van Esesn BC, Awwal AAS, Asari VK (2018) The history began from alexnet: A comprehensive survey on deep learning approaches. arXiv preprint arXiv:180301164

Avalos-López JI, Rojas-Domínguez A, OrnelasRodríguez M, Carpio M, Valdez SI (2021) Efficient training of deep learning models through improved adaptive sampling. In: Roman-Rangel E, KuriMorales ÁF, Martínez-Trinidad JF, Carrasco-Ochoa JA, Olvera-López JA (eds) Pattern Recognition, Springer International Publishing, Cham, pp 141-152

Bengio Y, Louradour J, Collobert R, Weston J (2009) Curriculum learning. In: Proceedings of the 26th annual international conference on machine learning, pp 41-48

Bouchard G, Trouillon T, Perez J, Gaidon A (2015) Online learning to sample. arXiv preprint arXiv:150609016

Fan Y, Tian F, Qin T, Bian J, Liu TY (2017) Learning what data to learn. arXiv preprint arXiv:170208635

Gopal S (2016) Adaptive sampling for sgd by exploiting side information. In: International Conference on Machine Learning, PMLR, pp 364-372

Joseph K, Singh K, Balasubramanian VN, et al. (2019) Submodular batch selection for training deep neural networks. arXiv preprint arXiv:190608771

Katharopoulos A, Fleuret F (2017) Biased importance sampling for deep neural network training. arXiv preprint arXiv:170600043

Katharopoulos A, Fleuret F (2018) Not all samples are created equal: Deep learning with importance sampling. In: International conference on machine learning, PMLR, pp 2525-2534 
Kawaguchi K, et al. (2020) On optimization and scalability in deep learning. PhD thesis, Massachusetts Institute of Technology

Krizhevsky A, Hinton G, et al. (2009) Learning multiple layers of features from tiny images. Tech. rep., University of Toronto, URL http://www.cs.toronto. edu/ kriz/cifar.html

LeCun Y, Bottou L, Bengio Y, Haffner P (1998) Gradient-based learning applied to document recognition. Proceedings of the IEEE 86(11):2278-2324

Loshchilov I, Hutter F (2015) Online batch selection for faster training of neural networks. arXiv preprint arXiv: 151106343

Owen AB (2013) Monte Carlo theory, methods and examples. URL https://statweb.stanford.edu/ owen $/ \mathrm{mc} /$

Simonyan K, Zisserman A (2015) Very deep convolutional networks for large-scale image recognition. 1409.1556

Smith SL, Kindermans PJ, Ying C, Le QV (2017) Don't decay the learning rate, increase the batch size. arXiv preprint arXiv: 171100489

Tokdar ST, Kass RE (2010) Importance sampling: a review. Wiley Interdisciplinary Reviews: Computational Statistics 2(1):54-60

Wang L, Ye J, Zhao Y, Wu W, Li A, Song SL, Xu Z, Kraska T (2018) Superneurons: Dynamic gpu memory management for training deep neural networks. In: Proceedings of the 23rd ACM SIGPLAN symposium on principles and practice of parallel programming, pp 41-53

Wang M, Fu W, He X, Hao S, Wu X (2020) A survey on large-scale machine learning. IEEE Transactions on Knowledge and Data Engineering

Wang X (2020) Example weighting for deep representation learning. PhD thesis, Queen's University Belfast

Wu CY, Manmatha R, Smola AJ, Krahenbuhl P (2017) Sampling matters in deep embedding learning. In: Proceedings of the IEEE International Conference on Computer Vision, pp 2840-2848

Zhao P, Zhang T (2014) Accelerating minibatch stochastic gradient descent using stratified sampling. arXiv preprint arXiv: 14053080

Zhao P, Zhang T (2015) Stochastic optimization with importance sampling for regularized loss minimization. In: international conference on machine learning, PMLR, pp 1-9 